\title{
«Il Re di pietra». Anna Frabetti dialoga con Erminia Dell'Oro
}

\section{Anna Frabetti e Erminia Dell'Oro}

\section{(2) OpenEdition}

1 Journals

\section{Edizione digitale}

URL: https://journals.openedition.org/cher/10769

DOI: 10.4000/cher.10769

ISSN: 2803-5992

\section{Editore}

Presses universitaires de Strasbourg

\section{Edizione cartacea}

Data di pubblicazione: 30 juin 2013

Paginazione: 23-26

ISBN: 978-2-35410-054-4

ISSN: 1968-035X

Notizia bibliografica digitale

Anna Frabetti e Erminia Dell'Oro, ««ll Re di pietra». Anna Frabetti dialoga con Erminia Dell'Oro», reCHERches [Online], 10 | 2013, online dal 08 février 2022, consultato il 10 février 2022. URL: http:// journals.openedition.org/cher/10769; DOl: https://doi.org/10.4000/cher.10769

\section{(c) (i) (8)}

Ce(tte) œuvre est mise à disposition selon les termes de la Licence Creative Commons Attribution Pas d'Utilisation Commerciale - Partage dans les Mêmes Conditions 4.0 International. 


\title{
«ll Re di pietra». Anna Frabetti dialoga con Erminia Dell'Oro
}

\author{
Anna Frabetti \\ Université de Strasbourg \\ Erminia DelL'Oro \\ Scrittrice
}

Anna Frabetti: Come e quando è approdata alla scrittura?

Erminia Dell'Oro: Ho sempre scritto, fin da bambina. Ero un'appassionata lettrice, e mi piaceva scrivere. Scrivevo racconti, impressioni, mai diari. Una mia insegnante di italiano, ad Asmara, mi diceva che sarei diventata una scrittrice. Mi ha rintracciato molti anni dopo a Milano, aveva letto Asmara addio. Voleva ricordarmi le sue parole. Il primo romanzo l'ho scritto a vent'anni, ero a Milano da pochi mesi e attraverso la scrittura combattevo la nostalgia di Asmara, il disorientamento. Non lo ha mai letto nessuno. È poi naufragato in una cantina, non è stata una grande perdita. Ho poi tentato la strada del giornalismo, studiando, ma per una ragazza che arrivava a Milano da una lontana ex colonia, e il maschilismo imperante di quegli anni non era facile. Ho iniziato a pubblicare libri circa venticinque anni fa.

A. F.: In quale modo si è compiuto il passaggio, per così dire, alla scrittura per bambini e per ragazzi, genere praticato da molti scrittori migranti italiani, tra l'altro?

E.D.: Il passaggio alla scrittura per bambini e per ragazzi è avvenuto in modo casuale. Non avevo mai pensato di scrivere per bambini. Un giorno sono andata al Museo di Storia Naturale di Milano con un mio nipotino 
di quattro anni. Lui si è incantato davanti a un triceratopo riprodotto a grandezza naturale. Quel dinosauro era diventato una sua fissazione, tornavamo spesso al Museo. In quei giorni ho scritto un racconto per lui, perché lo leggesse da adulto. Non pensavo di pubblicarlo. Un mio amico lo ha letto e lo ha mandato alla redazione dell'Einaudi ragazzi. Il racconto è stato subito pubblicato con il titolo Matteo e i dinosauri, e ha avuto successo. Ho chiesto a Matteo di suggerirmi un altro titolo. La pianta magica ha risposto, forse pensando ai miei racconti sull'Africa. Ho scritto La pianta magica, uno dei miei libretti più ristampati. Da allora non mi sono più fermata. Senza Matteo e il triceratopo non avrei mai scritto per bambini. In seguito ho scritto anche per ragazzi, libri ispirati a storie vere.

A. F.: Lei è stata da tempo annoverata fra le principali rappresentanti della letteratura postcoloniale italiana, una letteratura che ha acquisito negli ultimi anni una notevole visibilità, grazie ad un lavoro di riflessione storica, di autocoscienza, di recupero memoriale operato proprio dagli scrittori. Come si colloca lei in questa corrente e qual è il suo giudizio in merito?

E. D.: Il mio primo libro, Asmara addio, è uscito quando ancora non si accennava alla letteratura postcoloniale. Mi ero resa conto che insieme al ventennio fascista era stato rimosso il periodo coloniale. Avevo avuto modo, in Italia, di constatare quanto ignorate fossero le ex colonie, ancora oggi tanti italiani non sanno dove sia l'Eritrea. Per il capitolo in cui scrivo dell'aggressione all'Etiopia e dei bombardamenti con l'iprite sono stata insultata e ho ricevuto lettere anonime. Il mio libro ha avuto successo perché era, allora, una novità. Oggi, finalmente, altri scrittori, anche giovani, affrontano la letteratura postcoloniale, partendo anche da storie familiari. Il mio giudizio è, ovviamente, positivo, e mi sento una decana di questa corrente.

Sono nata in una famiglia di origine italiana, ho studiato nelle scuole italiane. Mio padre faceva parte di coloro che venivano definiti i «vecchi coloniali», da non confondersi con i tanti italiani arrivati nel 1936 con la tessera del fascismo. Mio padre era uno dei pochi antifascisti, e si muoveva, anche per ragioni di lavoro, in un ambiente cosmopolita, tenendo presente che Asmara è una piccola città. Frequentavamo famiglie indiane, arabe, greche, slave, mio padre giocava a scacchi con un maggiore polacco naturalizzato inglese. Le pochissime volte che si prendeva una vacanza la sua meta era lo Yemen. Donne, uomini e bambini eritrei hanno avuto, con le loro storie personali 
e quelle raccontate, molta influenza sulla mia crescita. La mia «lingua» non poteva che essere contaminata.

A. F.: Giuliana Benvenuti ha scritto recentemente, a proposito della produzione letteraria centrata sulla rielaborazione della storia coloniale italiana, che esiste una differenza sostanziale tra $i$ testi degli autori italiani e quelli degli autori migranti o figli di coppie miste:

"gli autori italiani raramente tematizzano la storia della resistenza verso il colonizzatore, mentre gli autori migranti di essa diffusamente si occupano, introducendo una doppia visione nel racconto della storia coloniale, che assume a pieno titolo una prospettiva postcoloniale»1.

Cosa ne pensa?

E.D.: Giuliana Benvenuti ha ragione. Finalmente vengono pubblicati libri, come il bel romanzo di Gabriella Ghermandi, in cui viene introdotta la doppia visione della storia coloniale. Ho spesso pensato alla "doppia visione", e nel 1999 ho scritto un romanzo dal titolo Il Re di pietra, riferendomi, con il titolo, all'obelisco di Axum portato via dagli italiani negli anni della guerra di aggressione all'Etiopia. Mi sono documentata sui testi del professor Angelo Del Boca e su testi di storici inglesi per non commettere errori nella stesura del libro. L'ho mandato al professor Del Boca per avere un suo giudizio e lui mi ha scritto una bella lettera in cui esprimeva il suo apprezzamento al libro, sicuro che sarebbe stato pubblicato. Lo ha letto il dottor Cerati, attuale presidente della Casa Editrice Einaudi e ha detto che secondo lui $\mathrm{Il} \mathrm{Re} d i$ Pietra era il mio libro migliore. Ma il libro non è stato pubblicato, né da Einaudi, né da altri editori ai quali lo avevo mandato. Sono trascorsi tredici anni, proverò ora a mandarlo a qualche altro editore. Nel romanzo, il cui protagonista è un ex cantastorie axumita alla corte dell'imperatore Hailè Selassiè, descrivo i crimini commessi dai militari, anche dai civili italiani, le rappresaglie terribili di Rodolfo Graziani, l'assassinio di centinaia di monaci, indovini, maestri, cantastorie, ed episodi dell'eroica resistenza dei Ras, tra i quali il valoroso Ras Immirù. Nonché i bombardamenti con l'iprite. Ho descritto questo episodio personale perché se Il Re di pietra fosse stato pubblicato allora sarei stata, come per Asmara addio, uno dei primi scrittori ad avere la doppia visione, contraddicendo la tesi di Giuliana Benvenuti.

1 G. Benvenuti, Letteratura della migrazione, letteratura postcoloniale, letteratura italiana. Problemi di definizione, in Leggere il testo e il mondo. Vent'anni di scritture della migrazione in Italia, a cura di F. Pezzarossa e I. Rossini, Bologna, Clueb, 2011, p. 252. 
A. F.: Se la potenziale riconciliazione con l'Italia e con il suo passato avviene nella lingua, nella scelta dell'italiano come lingua letteraria, il rapporto con la cultura e la letteratura italiana rimane forse ancora problematico. Gabriella Ghermandi, nel suo ultimo romanzo Regina di fiori e di perle, riscrive, come ha notato Roberto Derobertis, un passaggio di Tempo di uccidere di Flaiano, cosa del tutto nuova nella letteratura italiana migrante, ma non in altre letterature postcoloniali. Qual è la sua posizione in merito?

E. D.: In Italia per molti anni c'è stata una vergognosa rimozione. Soltanto quando sono stati aperti i cosiddetti armadi della vergogna ci si è trovati a dover fare i conti con la storia. Quella vera, ignorata per molto tempo anche nei testi scolastici. Il mito degli «italiani brava gente» fa ancora fatica a morire. Ricordo che qualcuno mi ha scritto, dopo avere letto il mio libro L'Abbandono: "gentile signora si ricordi che i panni sporchi si lavano in casa». Una frase molto significativa sulla mentalità degli italiani. Negli altri paesi i conti con la storia sono stati fatti prima, e si capisce dalle letterature che ne sono nate. Oggi, grazie al recupero della memoria, e a bravi scrittori migranti, o figli di coppie miste, si sta recuperando la nostra storia coloniale vista con il doppio sguardo. 